\title{
THE EULER SCHEME FOR A STOCHASTIC DIFFERENTIAL EQUATION DRIVEN BY PURE JUMP SEMIMARTINGALES
}

\author{
HANCHAO WANG, ${ }^{*}$ Zhejiang University
}

\begin{abstract}
In this paper we propose the asymptotic error distributions of the Euler scheme for a stochastic differential equation driven by Itô semimartingales. Jacod (2004) studied this problem for stochastic differential equations driven by pure jump Lévy processes and obtained quite sharp results. We extend his results to a more general pure jump Itô semimartingale.
\end{abstract}

Keywords: Euler scheme; weak convergence; pure jump Itô semimartingale; stochastic differential equation

2010 Mathematics Subject Classification: Primary 60J75; 65C30

Secondary 60J30; 60F17

\section{Introduction}

We consider the following stochastic differential equation (SDE):

$$
X_{t}=x_{0}+\int_{0}^{t} f\left(X_{s-}\right) \mathrm{d} Y_{s},
$$

where $f$ denotes a continuous function and $Y$ is the Itô semimartingale.

According to the equation

$$
X_{0}^{n}=x_{0}, \quad X_{i / n}^{n}=X_{(i-1) / n}^{n}+f\left(X_{(i-1) / n}^{n}\right)\left(Y_{i / n}-Y_{(i-1) / n}\right),
$$

the approximated solution of (1) is defined at the time $i / n$ by induction on the integer $i$. This scheme is called a Euler scheme. To find an approximation of the law of the path, we need to study the weak convergence of

$$
u_{n} U_{t}^{n}=u_{n}\left(X_{[n t] / n}^{n}-X_{[n t] / n}\right) .
$$

This problem has attracted the attention of a number of authors. The main gap in this problem is to find a sharp rate, $u_{n}$. Furthermore, this problem is the central topic in the asymptotic analysis of discretization of stochastic processes. In their monograph, Jacod and Protter [4] collected the recent developments of this field. It is worth noting that Jacod and Protter [3] provided some fundamental results on the asymptotic error distributions of SDEs, and studied the SDEs driven by continuous semimartingales and Lévy processes in detail. They gave the sharp rate as $u_{n}=\sqrt{n}$ when the continuous martingale part was present in Lévy processes. When the continuous martingale is not present then the results are quite different. Jacod [2] studied the SDEs driven by pure jump Lévy processes. He gave the rates in terms of the concentration of the Lévy measure.

Received 1 November 2013; revision received 25 February 2014.

* Postal address: Department of Mathematics, Zhejiang University, 38 Zheda Road, Hangzhou, 310027, China. Email address: hcwang06@gmail.com 
In this paper we aim to extend the results in Jacod [2] to more general Itô semimartingales. Jacod employed the independent and stationary properties of increments of Lévy processes to obtain the results. However, when we study the same problem for a more general Itô semimartingale, it is difficult to obtain similar results following the same approach. Recently, Diop et al. [1] studied the weak convergence of approximate quadrate variations toward the true quadratic variations of pure jump Itô semimartingales. This topic is very popular, especially in the study of mathematical finance. We draw support from the method in Diop et al. [1], and overcome the gap in the extension.

This paper is organized as follows. In Section 2 we express the main result. Some technical lemmas are presented in Section 3. We give the proof of the main result in Section 4.

\section{Main result}

A semimartingale $M$ is an Itô semimartingale on some filtered space $\left(\Omega, \mathcal{F},\left(\mathcal{F}_{t}\right)_{t \geq 0}, \mathbb{P}\right)$ if its characteristics $\left(B^{M}, C^{M}, v^{M}\right)$ are absolutely continuous with respect to the Lebesgue measure. In other words, the characteristics of $M$ have the form

$$
B_{t}^{M}=\int_{0}^{t} b_{s}^{M} \mathrm{~d} s, \quad C_{t}^{M}=\int_{0}^{t} c_{s}^{M} \mathrm{~d} s, \quad v^{M}(\mathrm{~d} t, \mathrm{~d} x)=\mathrm{d} t F_{t}^{M}(\mathrm{~d} x) .
$$

Here, $b^{M}$ and $c^{M}$ are optional processes, with $c^{M} \geq 0$, and $F^{M}$ is an optional random measure on $\mathbb{R}$. The triple $\left(b_{t}^{M}, c_{t}^{M}, F_{t}^{M}\right)$ constitutes the spot characteristics of $M$. The details of these concepts and notions can be found in Jacod and Shiryaev [5].

In this section we look at the common feature of our setting which is that the second characteristic of $Y$ vanishes identically. It means that the continuous local martingale part is not present in $Y$.

Assumption 1. We have

$$
Y_{t}=\int_{0}^{t} \sigma_{s-} \mathrm{d} Z_{s}
$$

where

(a) $Z$ is a nonhomogeneous Lévy process with (nonrandom) spot characteristics $\left(b_{t}^{Z}, 0, G_{t}\right)$, $b_{t}^{\prime Z}=b_{t}^{Z}-\int_{\{|x| \leq 1\}} x G_{t}(\mathrm{~d} x)$ and constant $\alpha \in(0,2)$, and two functions $\theta_{t}^{+}, \theta_{t}^{-} \geq 0$ on $\mathbb{R}_{+}$ such that,

$$
\lim _{x \downarrow 0} \sup _{0 \leq t \leq 1}\left|x^{\alpha} \bar{G}_{t}^{ \pm}(x)-\theta_{t}^{ \pm}\right|=0,
$$

where $\bar{G}_{t}^{+}(x)=G_{t}((x, \infty)), \bar{G}_{t}^{-}(x)=G_{t}((-\infty,-x)), b_{t}^{Z}$ is locally bounded, and $\theta_{t}^{+}, \theta_{t}^{-}$ are Riemann integrable over each finite interval.

(b) The process $\sigma$ is an Itô semimartingale with spot characteristics $\left(b_{t}^{\sigma}, c_{t}^{\sigma}, F_{t}^{\sigma}\right)$, such that the processes $b_{t}^{\sigma}, c_{t}^{\sigma}$ and $\int\left(x^{2} \wedge 1\right) F_{t}^{\sigma}(\mathrm{d} x)$ are locally bounded.

Assumption 2. We have Assumption 1, and

$$
\sup _{0 \leq t \leq 1} \int_{0}^{1}\left|\bar{G}_{t}^{+}(x)-\bar{G}_{t}^{-}(x)\right| \mathrm{d} x<\infty .
$$

Assumption 3. We have that $f$ is a $C^{3}$ (three times differentiable) function. Set $\bar{G}_{t}(x)=$ $\bar{G}_{t}^{+}(x)+\bar{G}_{t}^{-}(x), \theta=\theta^{+}+\theta^{-}, \theta^{\prime}=\theta^{+}-\theta^{-}$. 
Theorem 1. Under Assumption 3 and in the following cases, the sequence $\left(\bar{Y}^{n}, u_{n} U^{n}\right)$ converges in law to $(Y, U)$, where $U$ is the unique solution of the linear equation

$$
U_{t}=\int_{0}^{t} f^{\prime}\left(X_{s-}\right) U_{s-} \mathrm{d} Y_{s}-W_{t},
$$

and where $W$ can be described as follows.

Case I. Under Assumption 1 with $\alpha>1$, then $u_{n}=(n / \log n)^{1 / \alpha}$ and

$$
W_{t}=\int_{0}^{t} f\left(X_{s-}\right) f^{\prime}\left(X_{s-}\right) \sigma_{s-}^{2} \mathrm{~d} V_{s},
$$

where $V$ is another Lévy process, independent of $Z$, with spot characteristics $\left(b_{t}^{V}, 0, G_{t}^{V}\right)$ given by

$$
\begin{gathered}
b_{t}^{V}=\frac{-\alpha\left(\theta_{t}^{\prime}\right)^{2}}{2(\alpha-1)}, \\
G_{t}^{V}(\mathrm{~d} x)=\frac{\alpha}{2}\left[\left(\left(\theta_{t}^{+}\right)^{2}+\left(\theta_{t}^{-}\right)^{2}\right) \mathbf{1}_{\{x>0\}}+2 \theta_{t}^{+} \theta_{t}^{-} \mathbf{1}_{\{x<0\}}\right] \frac{1}{|x|^{1+\alpha}} \mathrm{d} x .
\end{gathered}
$$

Case II. Under Assumption 1 with $\alpha=1$, then $u_{n}=n /(\log n)^{2}$ and

$$
W_{t}=-\frac{1}{4} \int_{0}^{t} f\left(X_{s-}\right) f^{\prime}\left(X_{s-}\right) \sigma_{s-}^{2} \theta_{s}^{\prime 2} \mathrm{~d} s .
$$

Case III. Under Assumptions 1 and 2, $\alpha<1$ with $b_{t}^{\prime Z}=0$, or $\alpha=1$ then $u_{n}=(n / \log n)^{1 / \alpha}$, and

$$
W_{t}=\int_{0}^{t} f\left(X_{s-}\right) f^{\prime}\left(X_{s-}\right) \sigma_{s-}^{2} \mathrm{~d} V_{s},
$$

where $V$ is another Lévy process, independent of $Z$, with spot characteristics $\left(b_{t}^{V}, 0, G_{t}^{V}\right)$ given by

$$
b_{t}^{V}=0, \quad G_{t}^{V}(\mathrm{~d} x)=\frac{\theta_{t}^{2} \alpha}{4|x|^{1+\alpha}} \mathrm{d} x .
$$

Note that,

$$
\begin{aligned}
U_{t}^{n}= & \sum_{i=1}^{[n t]} \int_{(i-1) / n}^{i / n}\left(f\left(X_{(i-1) / n}^{n}\right)-f\left(X_{(i-1) / n}\right)\right) \mathrm{d} Y_{s} \\
& -\sum_{i=1}^{[n t]} \int_{(i-1) / n}^{i / n}\left(f\left(X_{s-}\right)-f\left(X_{(i-1) / n}\right)\right) \mathrm{d} Y_{s},
\end{aligned}
$$

and set $\bar{Y}_{t}^{n}=Y_{[n t] / n}, \bar{X}_{t}^{n}=X_{[n t] / n}$, and

$$
W_{t}^{n}=\sum_{i=1}^{[n t]} \int_{(i-1) / n}^{i / n}\left(f\left(X_{s-}\right)-f\left(X_{(i-1) / n}\right)\right) \mathrm{d} Y_{s} .
$$

We obtain

$$
U_{t}^{n}=\int_{0}^{t}\left(f\left(\bar{X}_{s-}^{n}+U_{s-}^{n}\right)-f\left(\bar{X}_{s-}^{n}\right)\right) \mathrm{d} \bar{Y}_{s}^{n}-W_{t}^{n} .
$$

Based on (13), and Theorem 2.2 of Jacod [2], it is enough to prove

$$
\left(\bar{Y}^{n}, u_{n} W^{n}\right) \quad \Longrightarrow \quad(Y, W)
$$

to obtain Theorem 1 . 
Remark 1. Theorem 2.2 of Jacod [2] can be obtained directly from Theorem 3.2 of Jacod and Protter [3], which itself is based upon Kurtz and Protter [6]. Theorem 3.2 of Jacod and Protter [3] concerns the general semimartingale, so it can also work for $Y$ in this paper.

In the following, we will focus on proving (14) under the conditions of Theorem 1.

\section{Technical lemmas}

In this section we present technical lemmas in order to prove (14).

\subsection{Localization}

Our task in this subsection is to avoid a lot of technical problems. We will reduce the problem to a situation where $f$ and $Y$ satisfy some strengthened version of our assumptions, which are as follows.

Assumption S1. We have Assumption 1, and, moreover,

(a) $\left|\Delta Z_{t}\right|$ and $\left|\sigma_{t}\right|$ are bounded for all $t \in[0,1]$;

(b) we have $\sup _{x>0, t \geq 0} x^{\alpha} \overline{G_{t}}(x)<\infty$.

(c) the processes $\left|b_{t}^{\sigma}\right|, c_{t}^{\sigma}$, and $\int x^{2} F_{t}^{\sigma}(\mathrm{d} x)$ are bounded.

(d) $\lim _{z \rightarrow 0} \phi(z)=\lim _{z \rightarrow 0} \sup _{t \geq 0}\left(\left|z^{\alpha} \bar{G}_{t}^{+}(z)-\theta_{t}^{+}\right|+\left|z^{\alpha} \bar{G}_{t}^{-}(z)-\theta_{t}^{-}\right|\right)=0$.

Assumption S2. We have Assumptions 2 and S1 with $\alpha=1$.

Assumption S3. We have that $f$ is a $C^{3}$ (three times differentiable) function with compact support.

Lemma 1. In Theorem 1, one can replace Assumption 1, 2, or 3 with Assumption S1, S2, or S3.

Proof. We suppose Theorem 1 holds under Assumptions S1, S2, and S3. We need to prove that Theorem 1 still holds under Assumptions 1, 2, and 3.

Consider the new Lévy process $Z(p)_{t}=Z_{t}-\sum_{s \leq t} \Delta Z_{s} \mathbf{1}_{\left\{\left|\Delta Z_{s}\right| \geq p\right\}}$, where $\mathbf{1}$ is the indicator function and a $C^{3}$ function $f_{p}$ with compact support, satisfying $f_{p}(x)=f(x)$ for $|x| \leq p^{2}$. Similar to Jacod [2], associate with $Z(p)$ and $f_{p}$ the same term with $Z$ and $f$, writing $X(p)$, $\bar{Y}(p)^{n}, U(p)^{n}$ instead of $X, \bar{Y}^{n}, U^{n}$

By hypothesis, we have a sequence of stopping times $\left(\tau_{p}\right)_{p \geq 1}$, and a sequence of nonrandom times $\left(t_{p}\right)_{p \geq 1}$, with $\tau_{p} \leq t_{p}$, and $\tau_{p} \uparrow \infty$ as $p \rightarrow \infty$ such that

$$
\sup _{x \in(0,1]} x^{\alpha} \bar{G}_{t}(x) \leq p, \quad\left|b_{t}^{Z}\right| \leq p, \quad \int_{0}^{1}\left|\bar{G}_{t}^{+}(x)-\bar{G}_{t}^{-}(x)\right| \mathrm{d} x \leq p
$$

when $t \leq t_{p}$,

$$
\left|b_{t}^{\sigma}\right| \leq p, \quad c_{t}^{\sigma} \leq p, \quad\left|\sigma_{t}\right| \leq p, \quad \int\left(x^{2} \wedge 1\right) F_{t}^{\sigma}(\mathrm{d} x) \leq p, \quad|\Delta Z| \leq p
$$

when $t \leq \tau_{p}$. Set $\sigma(p)_{t}=\sigma_{t \wedge \tau_{p}}, Y(p)_{t}=\int_{0}^{t} \sigma(p)_{s-} \mathrm{d} Z(p)_{s}$, and $\theta_{t}^{ \pm}(p)=\theta_{t}^{ \pm} \mathbf{1}_{\left\{t \leq t_{p}\right\}}$. We easily conclude that

$$
t \leq \tau_{p} \quad \Longrightarrow \quad \sigma(p)_{t}=\sigma, \quad Z(p)_{t}=Z, \quad Y(p)=Y .
$$


Our hypothesis now implies that $\left(\bar{Y}^{n}(p), u_{n} U^{n}(p)\right)$ converges in law to $(Y(p), U(p))$ with $\theta^{ \pm}(p)$ instead of $\theta^{ \pm}, f_{p}$ instead of $f$, and $\sigma(p)$ instead of $\sigma$. We see that the restriction of $U$ to $\left[0, \tau_{p}\right)$ is a version of the restriction of $U(p)$ to $\left[0, \tau_{p}\right)$.

For any continuous bounded function $\Phi_{t}$ on the Skorokhod space $\mathbb{D}\left(\mathbb{R}_{+}, \mathbb{R}^{2}\right)$, which depends on the sample path only up to time $t$, we have

$$
\begin{gathered}
\left|\mathbb{E}\left(\Phi_{t}\left(\bar{Y}^{n}, u_{n} U^{n}\right)\right)-\mathbb{E}\left(\Phi_{t}\left(\bar{Y}^{n}(p), u_{n} U^{n}(p)\right)\right)\right| \leq 2\left\|\Phi_{t}\right\| \mathbb{P}\left(\tau_{p} \leq t\right), \\
\left|\mathbb{E}\left(\Phi_{t}(Y, U)\right)-\mathbb{E}\left(\Phi_{t}(Y(p), U(p))\right)\right| \leq 2\left\|\Phi_{t}\right\| \mathbb{P}\left(\tau_{p} \leq t\right) .
\end{gathered}
$$

Since $\mathbb{P}\left(\tau_{p} \leq t\right) \rightarrow 0$ and

$$
\mathbb{E}\left(\Phi_{t}\left(\bar{Y}^{n}(p), u_{n} U^{n}(p)\right)\right) \rightarrow \mathbb{E}\left(\Phi_{t}(Y(p), U(p))\right)
$$

for every $t$ as $p \rightarrow \infty$, we obtain this lemma.

\subsection{Estimates of nonhomogeneous Lévy processes}

Now we split the processes $Z, Y$, and $H=f(X)$. We recall that $\mu^{Z}$ is the jump measure of $Z$ and $v^{Z}(\mathrm{~d} s, \mathrm{~d} x)=G_{s}(\mathrm{~d} x) \mathrm{d} s$ is its predictable compensator.

Let $0<v<1$, set

$$
Z=A^{v}+M^{v}+N^{v}
$$

where $A^{v}=b^{Z}-x \mathbf{1}_{\{v \leq|x| \leq 1\}} * v^{Z}, M^{v}=x \mathbf{1}_{\{|x| \leq v\}} *\left(\mu^{Z}-v^{Z}\right)$, and $N^{v}=x \mathbf{1}_{\{|x|>v\}} * \mu^{Z}$, and

$$
Y=\widetilde{A}^{v}+\tilde{M}^{v}+\tilde{N}^{v},
$$

where $\widetilde{A}_{t}^{v}=\int_{0}^{t} \sigma_{s-} \mathrm{d} A_{s}^{v}, \tilde{M}_{t}^{v}=\int_{0}^{t} \sigma_{s-} \mathrm{d} M_{s}^{v}$, and $\tilde{N}_{t}^{v}=\int_{0}^{t} \sigma_{s-} \mathrm{d} N_{s}^{v}$.

Let $g$ be $f f^{\prime}$, there is the decomposition

$$
G(x, y):=f(x+y f(x))-f(x)=y g(x)+y^{2} k(x, y),
$$

and, furthermore, we have decompositions of the semimartingale $H=f(X)$,

$$
H=\widetilde{A}^{\prime} v+\widetilde{M}^{\prime} v+\widetilde{N}^{\prime} v,
$$

where

$$
\begin{gathered}
\widetilde{A}^{\prime} v=g\left(X_{t-}\right) \widetilde{A}^{v}+k\left(X_{t-}, \sigma_{t-} x\right)\left(\sigma_{t-} x\right)^{2} \mathbf{1}_{\{|x| \leq v\}} * v^{Z}, \\
\widetilde{M}^{\prime}{ }^{v}=G\left(X_{t-}, \sigma_{t-} x\right) \mathbf{1}_{\{|x| \leq v\}} *\left(\mu^{Z}-v^{Z}\right),
\end{gathered}
$$

and

$$
\widetilde{N}^{v}=G\left(X_{t-}, \sigma_{t-} x\right) \mathbf{1}_{\{|x|>v\}} * \mu^{Z} .
$$

Furthermore, set

$$
Z^{v}=A^{v}+M^{v}, \quad Y^{v}=\widetilde{A}^{v}+\widetilde{M}^{v}, \quad H^{v}={\widetilde{A^{\prime}}}^{v}+{\widetilde{M^{\prime}}}^{v} .
$$

To prove (14), we need to introduce a sequence $v_{n} \rightarrow 0$, from the idea of Jacod [2].

$$
\text { Case I: } v_{n}=\frac{\log n}{n^{1 /(2 \alpha)}}, \quad \text { Case II: } v_{n}=\frac{\log n}{n}, \quad \text { Case III: } v_{n}=\left(\frac{\log n}{n}\right)^{1 / \alpha} \text {. }
$$

For any stochastic process $Q_{t}$, we define $Q_{t}^{n, i}=Q_{t}^{v_{n}}-Q_{(i-1) / n}^{v_{n}}$ for $t \geq(i-1) / n$ such that

$$
V_{t}^{n}(i)=G\left(X_{(i-1) / n}, \sigma_{(i-1) / n} x\right) \mathbf{1}_{\left\{|x| \geq v_{n}\right\}} \mathbf{1}_{((i-1) / n, \infty)}(s) * \mu_{T(n, i)_{1} \wedge t}^{Z} .
$$


Denote the successive jump times of $Z$, after $(i-1) / n$ and of size larger than or equal to $v_{n}$, by $T(n, i)_{p}$. Let $K(n, i)$ be the integer such that $T(n, i)_{K(n, i)} \leq i / n<T(n, i)_{K(n, i)+1}$.

- The times $T(n, i)$ forms a nonhomogeneous Poisson process with intensity $\bar{G}_{t}\left(v_{n}\right)$, and set $\alpha_{s, t}^{n}=\exp \left(-\int_{s}^{t} \bar{G}_{r}\left(v_{n}\right) \mathrm{d} r\right)$.

- Conditionally on $\mathscr{H}_{0}^{v_{n}}$ and $T(n, i)_{p}<\infty,\left(\Delta Z_{T(n, i)_{j}}\right)_{1 \leq j \leq p}$ are independent, with respective laws $\left(1 / \bar{G}_{T(n, i)_{j}}\left(v_{n}\right)\right) G_{T(n, i)_{j}}(\mathrm{~d} x) \mathbf{1}_{\left\{|x| \geq v_{n}\right\}}$.

Denote

$$
\begin{array}{rlrl}
c_{t}(v) & =\int_{\{|x| \leq v\}} x^{2} G_{t}(\mathrm{~d} x), & d_{t}^{+}(v) & =\int_{\{x>v\}}|x| G_{t}(\mathrm{~d} x), \\
d_{t}^{-}(v) & =\int_{\{x<-v\}}|x| G_{t}(\mathrm{~d} x), & \rho_{t}^{+}(v) & =\int_{\{x>v\}}|x|^{\alpha} G_{t}(\mathrm{~d} x), \\
\rho_{t}^{-}(v) & =\int_{\{x<-v\}}|x|^{\alpha} G_{t}(\mathrm{~d} x), & d_{t}^{\prime}(v) & =d_{t}^{+}(v)-d_{t}^{-}(v), \\
b_{t}^{\prime} & =b_{t}^{Z}+\int_{\{|x|>1\}} x G_{t}(\mathrm{~d} x), & \delta_{t}(v) & =d_{t}^{+}(v)+d_{t}^{-}(v), \\
d_{t}(v) & =b_{t}^{\prime}-d_{t}^{\prime}(v) .
\end{array}
$$

Lemma 2. If $1 \leq \alpha<p \leq 2$ or $\alpha<p \leq 1$,

$$
\mathbb{E}\left(\sup _{s \leq t}\left|\int_{T}^{T+s} H_{r} \mathrm{~d} M_{r}^{v}\right|^{p} \mid g_{T}^{v}\right) \leq K v^{p-\alpha} \mathbb{E}\left(\int_{T}^{T+s}\left|H_{r}\right|^{p} \mathrm{~d} r \mid g_{T}^{v}\right) .
$$

Lemma 3. Under the Assumptions $1-3$, with $\alpha \in(0,2)$, let $J_{r}$ be a random variable satisfying that $J_{r}, \quad K(n, i)$, and $\Delta Z_{T(n, i)_{r}}$ are independent conditionally on $\mathcal{F}_{(i-1) / n}$, and $\left|J_{r}\right| \leq K \sum_{j=1}^{r-1}\left|\Delta Z_{T(n, i)_{j}}\right|$, where $r \geq 2$. Let $J_{r}^{\prime}=J_{r} \Delta Z_{T(n, i)_{r}}$ for all $r^{\prime} \geq r \geq 2$ and $y>0$, we have

$$
\begin{aligned}
& \mathbb{P}\left(\left|u_{n} J_{r}^{\prime}\right|>y, K(n, i)=r^{\prime} \mid \mathcal{F}_{(i-1) / n}\right) \leq \frac{K}{\left(v_{n}^{\alpha}\right)^{r^{\prime}-2} n^{r^{\prime}}} \frac{(r-1)^{\alpha+1} u_{n}^{\alpha}}{y^{\alpha}}\left(1+\log \frac{1}{v_{n}}\right), \\
& \mathbb{E}\left(\left|u_{n} J_{r}\right|^{\alpha} \mathbf{1}_{\left\{K(n, i)=r^{\prime}\right\}} \mid \mathcal{F}_{(i-1) / n}\right) \leq \frac{K u_{n}^{\alpha} r^{2}\left(1+\log \left(1 / v_{n}\right)\right)}{\left(v_{n}^{\alpha}\right)^{r^{\prime}-1} n^{r^{\prime}}}, \\
& \mathbb{E}\left(\left|u_{n} J_{r}^{\prime}\right|^{2} \mathbf{1}_{\left\{\left|u_{n} J_{r}^{\prime}\right| \leq y \mid, K(n, i)=r^{\prime}\right\}} \mid \mathcal{F}_{(i-1) / n}\right) \leq \frac{K y^{2-\alpha} u_{n}^{\alpha} r^{2}\left(1+\log \left(1 / v_{n}\right)\right)}{\left(v_{n}^{\alpha}\right)^{r^{\prime}-2} n^{r^{\prime}}}, \\
& \mathbb{E}\left(\left|u_{n} J_{r}^{\prime}\right| \mathbf{1}_{\left\{\mid K(n, i)=r^{\prime}\right\}} \mid \mathcal{F}_{(i-1) / n}\right) \leq K r \frac{(\log n)^{2}}{\left(v_{n}^{\alpha}\right)^{r^{\prime}-1} n^{r^{\prime}}}, \\
& \alpha>1 \Longrightarrow \mathbb{E}\left(\left|u_{n} J_{r}^{\prime}\right| \mathbf{1}_{\left\{\left|u_{n} J_{r}^{\prime}\right|>y \mid, K(n, i)=r^{\prime}\right\}} \mid \mathcal{F}_{(i-1) / n}\right) \leq \frac{K u_{n}^{\alpha} r^{2}\left(1+\log \left(1 / v_{n}\right)\right)}{y^{\alpha-1}\left(v_{n}^{\alpha}\right)^{r^{\prime}-2} n^{r^{\prime}}}, \\
& \alpha<1 \Longrightarrow \mathbb{E}\left(\left|u_{n} J_{r}^{\prime}\right| \mathbf{1}_{\left\{\left|u_{n} J_{r}^{\prime}\right| \leq y \mid, K(n, i)=r^{\prime}\right\}} \mid \mathcal{F}_{(i-1) / n}\right) \leq \frac{K y^{1-\alpha} u_{n}^{\alpha} r^{2}\left(1+\log \left(1 / v_{n}\right)\right)^{2}}{\left(v_{n}^{\alpha}\right)^{r^{\prime}-2} n^{r^{\prime}}}
\end{aligned}
$$


Proof. We use the idea of Diop et al. [1] in the proof of this lemma. In fact, we need to associate the two filtrations $\left(\mathscr{g}_{t}^{v}\right)$ and $\left(\mathscr{H}_{t}^{v}\right)$, which are the smallest ones containing $\left(\mathcal{F}_{t}\right)$, such that

- $N_{t}^{v}$ is $g_{0}^{v}$-measurable for all $t$;

- $\sum_{s \leq t} \mathbf{1}_{\left\{\left|\Delta Z_{s}\right|>v\right\}}$ is $\mathscr{H}_{0}^{v}$-measurable for all $t$.

It is necessary to use these two filtrations in the argument, but, for simplicity, we do not present them in the following (see [1] for more details).

The left-hand side of (15) is less than or equal to

$$
\begin{aligned}
& \int_{\left\{s_{0}<s_{1}<\cdots<s_{r^{\prime}}<i / n\right\}} \prod_{l=1}^{r^{\prime}} \alpha_{s_{l-1}, s_{l}}^{n} \bar{G}_{s_{l}}\left(v_{n}\right) \mathbb{P}\left(\left|u_{n} J_{r}^{\prime}\right|>y \mid \mathcal{F}_{(i-1) / n}\right) \mathrm{d} s_{1} \mathrm{~d} s_{2} \cdots \mathrm{d} s_{r^{\prime}} \\
& \leq \int_{\left\{s_{0}<s_{1}<\cdots<s_{r^{\prime}}<i / n\right\}} \prod_{l=1}^{r^{\prime}} \alpha_{s_{l-1}, s_{l}}^{n} \prod_{l=1, l \neq r}^{r^{\prime}} \bar{G}_{s_{l}}\left(v_{n}\right) \\
& \times \int_{\left\{|x|>v_{n}\right\}} \mathbb{P}\left(\sum_{j=1}^{r-1}\left|\Delta Z_{T(n, i)_{j}}\right|>\frac{y}{u_{n} K x} \mid \mathcal{F}_{(i-1) / n}\right) G_{s_{r}}(\mathrm{~d} x) \mathrm{d} s_{1} \mathrm{~d} s_{2} \cdots \mathrm{d} s_{r^{\prime}} \\
& \leq \sum_{j=1}^{r-1} \int_{\left\{s_{0}<s_{1}<\cdots<s_{r^{\prime}}<i / n\right\}} \prod_{l=1}^{r^{\prime}} \alpha_{s_{l-1}, s_{l}}^{n} \prod_{l=1, l \neq r}^{r^{\prime}} \bar{G}_{s_{l}}\left(v_{n}\right) \\
& \times \int_{\left\{|x|>v_{n}\right\}} \mathbb{P}\left(\left|\Delta Z_{T(n, i)_{j}}\right|>\frac{y}{(r-1) u_{n} K x} \mid \mathcal{F}_{(i-1) / n}\right) G_{s_{r}}(\mathrm{~d} x) \mathrm{d} s_{1} \mathrm{~d} s_{2} \cdots \mathrm{d} s_{r^{\prime}} \\
& \leq \sum_{j=1}^{r-1} \int_{\left\{s_{0}<s_{1}<\cdots<s_{r^{\prime}}<i / n\right\}} \prod_{l=1}^{r^{\prime}} \alpha_{s_{l-1}, s_{l}}^{n} \prod_{l=1, l \neq r, l \neq j}^{r^{\prime}} \bar{G}_{s_{l}}\left(v_{n}\right) \\
& \times \int_{\left\{|x|>v_{n}\right\}} \mathbb{E}\left(\int \mathbf{1}_{\left\{|z|>y /(r-1) u_{n} K x\right\}} G_{S_{j}}(\mathrm{~d} z) \mid \mathcal{F}_{(i-1) / n}\right) G_{s_{r}}(\mathrm{~d} x) \mathrm{d} s_{1} \mathrm{~d} s_{2} \cdots \mathrm{d} s_{r^{\prime}} \\
& \leq \sum_{j=1}^{r-1} \frac{K}{\left(v_{n}^{\alpha}\right)^{r^{\prime}-2} n^{r^{\prime}}} \frac{(r-1)^{\alpha} u_{n}^{\alpha}}{y^{\alpha}}\left(1+\log \frac{1}{v_{n}}\right) \\
& \leq \frac{K}{\left(v_{n}^{\alpha}\right)^{r^{\prime}-2} n^{r^{\prime}}} \frac{(r-1)^{\alpha+1} u_{n}^{\alpha}}{y^{\alpha}}\left(1+\log \frac{1}{v_{n}}\right) .
\end{aligned}
$$

Second,

$$
\begin{aligned}
& \mathbb{E}\left(\left|J_{r}\right|^{\alpha} \mathbf{1}_{\left\{K(n, i)=r^{\prime}\right\}} \mid \mathcal{F}_{(i-1) / n}\right) \\
& \leq \int_{\left\{s_{0}<s_{1}<\cdots<s_{\left.r^{\prime}<i / n\right\}}\right.} \prod_{l=1}^{r^{\prime}} \alpha_{s_{l-1}, s_{l}}^{n} \bar{G}_{s_{l}}\left(v_{n}\right) \mathbb{E}\left(\left|\sum_{j=1}^{r-1} \Delta Z_{T(n, i) j}\right|^{\alpha} \mid \mathcal{F}_{(i-1) / n}\right) \mathrm{d} s_{1} \mathrm{~d} s_{2} \cdots \mathrm{d} s_{r^{\prime}} \\
& \leq(r-1) \sum_{j=1}^{r-1} \int_{\left\{s_{0}<s_{1}<\cdots<s_{r^{\prime}}<i / n\right\}} \\
& \quad \times \prod_{l=1}^{r^{\prime}} \alpha_{s_{l-1}, s_{l}}^{n} \bar{G}_{s_{l}}\left(v_{n}\right) \mathbb{E}\left(\left|Z_{T(n, i) j}\right|^{\alpha} \mid \mathcal{F}_{(i-1) / n}\right) \mathrm{d} s_{1} \mathrm{~d} s_{2} \cdots \mathrm{d} s_{r^{\prime}}
\end{aligned}
$$




$$
\begin{aligned}
& \leq(r-1) \sum_{j=1}^{r-1} \int_{\left\{s_{0}<s_{1}<\cdots<s_{r^{\prime}}<i / n\right\}} \prod_{l=1}^{r^{\prime}} \alpha_{s_{l-1}, s_{l}}^{n} \prod_{l=1, l \neq j}^{r^{\prime}} \bar{G}_{s_{l}}\left(v_{n}\right) \\
& \quad \times \int_{\left\{|x| \geq v_{n}\right\}}|x|^{\alpha} G_{s_{j}}(\mathrm{~d} x) \mathrm{d} s_{1} \mathrm{~d} s_{2} \cdots \mathrm{d} s_{r^{\prime}} \\
& \leq(r-1) \sum_{j=1}^{r-1} \frac{K\left(1+\log \left(1 / v_{n}\right)\right)}{\left(v_{n}^{\alpha}\right)^{r^{\prime}-1} n^{r^{\prime}}} \\
& \leq \frac{K r^{2}\left(1+\log \left(1 / v_{n}\right)\right)}{\left(v_{n}^{\alpha}\right)^{r^{\prime}-1} n^{r^{\prime}}} .
\end{aligned}
$$

Third,

$$
\begin{aligned}
\mathbb{E}\left(\left|u_{n} J_{r}^{\prime}\right|^{2} \mathbf{1}_{\left\{\left|u_{n} J_{r}^{\prime}\right| \leq y \mid, K(n, i)=r^{\prime}\right\}} \mid \mathcal{F}_{(i-1) / n}\right) \\
\quad=\mathbb{E}\left(\left|u_{n} J_{r}\right|^{2}\left|\Delta Z_{T(n, i)_{r}}\right|^{2} \mathbf{1}_{\left\{\left|\Delta Z_{T(n, i) r}\right| \leq y / \mid u_{n} J_{r} \|, K(n, i)=r^{\prime}\right\}} \mid \mathcal{F}_{(i-1) / n}\right) \\
\quad \leq \mathbb{E}\left(\left|u_{n} J_{r}\right|^{2} \mathbf{1}_{\left\{K(n, i)=r^{\prime}\right\}} \int_{(i-1) / n}^{i / n} \int_{\left\{|x| \leq y /\left|u_{n} J_{r}\right|\right\}} \frac{1}{\bar{G}_{S_{r}}\left(v_{n}\right)} x^{2} G_{s_{r}}(\mathrm{~d} x) \mathrm{d} s_{r} \mid \mathcal{F}_{(i-1) / n}\right) \\
\quad \leq K y^{2-\alpha} v_{n}^{\alpha} \mathbb{E}\left(\left|u_{n} J_{r}\right|^{\alpha} \mathbf{1}_{\left\{K(n, i)=r^{\prime}\right\}} \mid \mathcal{F}_{(i-1) / n}\right) \\
\quad \leq \frac{K y^{2-\alpha} u_{n}^{\alpha} r^{2}\left(1+\log \left(1 / v_{n}\right)\right)}{\left(v_{n}^{\alpha}\right)^{r^{\prime}-2} n^{r^{\prime}}},
\end{aligned}
$$

and

$$
\begin{aligned}
\mathbb{E}\left(\left|u_{n} J_{r}^{\prime}\right| \mathbf{1}_{\left\{\mid K(n, i)=r^{\prime}\right\}} \mid \mathcal{F}_{(i-1) / n}\right) & \leq \sum_{j=1}^{r-1} \mathbb{E}\left(\left|u_{n} \Delta Z_{T(n, i){ }_{j}} \Delta Z_{T(n, i)_{r}}\right| \mathbf{1}_{\left\{\mid K(n, i)=r^{\prime}\right\}} \mid \mathcal{F}_{(i-1) / n}\right) \\
& \leq K r \frac{(\log n)^{2}}{\left(v_{n}^{\alpha}\right)^{r^{\prime}-1} n^{r^{\prime}}}
\end{aligned}
$$

Finally, when $\alpha>1$,

$$
\begin{aligned}
& \mathbb{E}\left(\left|u_{n} J_{r}^{\prime}\right| \mathbf{1}_{\left\{\left|u_{n} J_{r}^{\prime}\right|>y \mid, K(n, i)=r^{\prime}\right\}} \mid \mathcal{F}_{(i-1) / n}\right) \\
& \quad=\mathbb{E}\left(\left|u_{n} J_{r}\right|\left|\Delta Z_{T(n, i)_{r}}\right| \mathbf{1}_{\left\{\left|\Delta Z_{T(n, i) r}\right|>y /\left|u_{n} J_{r}\right| \mid, K(n, i)=r^{\prime}\right\}} \mid \mathcal{F}_{(i-1) / n}\right) \\
& \quad \leq \mathbb{E}\left(\left|u_{n} J_{r}\right| \mathbf{1}_{\left\{K(n, i)=r^{\prime}\right\}} \int_{(i-1) / n}^{i / n} \int_{\left\{|x|>y /\left|u_{n} J_{r}\right|\right\}} \frac{1}{\bar{G}_{s_{r}}\left(v_{n}\right)}|x| G_{S_{r}}(\mathrm{~d} x) \mathrm{d} s_{r} \mid \mathcal{F}_{(i-1) / n}\right) \\
& \quad \leq \frac{K v_{n}^{\alpha}}{y^{\alpha-1}} \mathbb{E}\left(\left|u_{n} J_{r}\right|^{\alpha} \mathbf{1}_{\left\{K(n, i)=r^{\prime}\right\}} \mid \mathcal{F}_{(i-1) / n}\right) \\
& \quad \leq \frac{K u_{n}^{\alpha} r^{2}\left(1+\log \left(1 / v_{n}\right)\right)}{y^{\alpha-1}\left(v_{n}^{\alpha}\right)^{r^{\prime}-2} n^{r^{\prime}}},
\end{aligned}
$$

and when $\alpha<1$,

$$
\begin{aligned}
& \mathbb{E}\left(\left|u_{n} J_{r}^{\prime}\right| \mathbf{1}_{\left\{\left|u_{n} J_{r}^{\prime}\right| \leq y \mid, K(n, i)=r^{\prime}\right\}} \mid \mathcal{F}_{(i-1) / n}\right) \\
& \quad=\mathbb{E}\left(\left|u_{n} J_{r} \| \Delta Z_{T(n, i)_{r}}\right| \mathbf{1}_{\left\{\left|\Delta Z_{T(n, i) r}\right| \leq y / \mid u_{n} J_{r} \|, K(n, i)=r^{\prime}\right\}} \mid \mathcal{F}_{(i-1) / n}\right) \\
& \quad \leq \mathbb{E}\left(\left|u_{n} J_{r}\right|\left|\Delta Z_{T(n, i)_{r}}\right|^{\alpha} \frac{y^{1-\alpha}}{\left|u_{n} J_{r}\right|^{1-\alpha}} \mid \mathcal{F}_{(i-1) / n}\right)
\end{aligned}
$$




$$
\begin{aligned}
& \leq \frac{K y^{1-\alpha} v_{n}^{\alpha}}{n}\left(1+\log \left(\frac{1}{v_{n}}\right)\right) \mathbb{E}\left(\left|u_{n} J_{r}\right|^{\alpha} \mathbf{1}_{\left\{K(n, i)=r^{\prime}\right\}} \mid \mathcal{F}_{(i-1) / n}\right) \\
& \leq \frac{K y^{1-\alpha} u_{n}^{\alpha} r^{2}\left(1+\log \left(1 / v_{n}\right)\right)^{2}}{\left(v_{n}^{\alpha}\right)^{r^{\prime}-2} n^{r^{\prime}}}
\end{aligned}
$$

\subsection{Some key lemmas}

We will need the following lemmas to prove (14).

Lemma 4. For each $n>0$, every $\xi_{i}^{n}$ are $\mathcal{F}_{i / n}$-measurable random variables, and $a_{n}, a_{n}^{\prime}, a_{n, y}^{\prime \prime}$ are finite constants. Furthermore,

$$
\lim _{n \rightarrow \infty} a_{n}=0, \quad \lim _{n \rightarrow \infty} a_{n}^{\prime}=0, \quad \lim _{n \rightarrow \infty} a_{n, y}^{\prime \prime}=0 \quad \text { for all } y>1 .
$$

If one of the following conditions hold:

(i)

$$
\mathbb{E}\left[\left|\xi_{i}^{n}\right| \mid \mathcal{F}_{(i-1) / n}\right] \leq \frac{a_{n}}{n},
$$

(ii)

$$
\begin{gathered}
\left|\mathbb{E}\left[\xi_{i}^{n} \mid \mathcal{F}_{(i-1) / n}\right]\right| \leq \frac{a_{n}}{n}, \quad \mathbb{E}\left[\left|\xi_{i}^{n}\right|^{2} \mid \mathcal{F}_{(i-1) / n}\right] \leq \frac{a_{n}^{\prime}}{n}, \\
\left|\mathbb{E}\left[\xi_{i}^{n} \mathbf{1}_{\left\{\left|\xi_{i}^{n}\right| \leq 1\right\}} \quad \mid \mathcal{F}_{(i-1) / n}\right]\right| \leq \frac{a_{n}}{n},
\end{gathered}
$$

(iii)

$$
\mathbb{E}\left[\left|\xi_{i}^{n}\right|^{2} \mathbf{1}_{\left\{\left|\xi_{i}^{n}\right| \leq 1\right\}} \mid \mathcal{F}_{(i-1) / n}\right] \leq \frac{a_{n}^{\prime}}{n}, \quad \mathbb{P}\left[\left|\xi_{i}^{n}\right|>y \mid \mathcal{F}_{(i-1) / n}\right] \leq \frac{a_{n, y}^{\prime \prime}}{n} \quad \text { for all } y>1,
$$

then

$$
\sup _{s \leq t}\left|\sum_{i=1}^{[n s]} \xi_{i}^{n}\right| \stackrel{\mathbb{P}}{\rightarrow} 0
$$

In the following, for the stochastic process $\Gamma=\left(\Gamma_{t}\right)_{t \geq 0}$, we denote $\sup _{s \leq t}\left|\Gamma_{s}\right| \stackrel{\mathbb{P}}{\rightarrow} 0$ by $\Gamma \stackrel{\text { u.c.p. }}{\longrightarrow} 0$, where 'u.c.p.' denotes uniform convergence in probability,

$$
\begin{aligned}
\Delta^{n}(j)_{t} & =\sum_{i=1}^{[n t]} \xi_{i}^{n}(j), \\
\xi_{i}^{n}(1) & =u_{n} \int_{I(n, i)}\left(H_{s-}^{n, i}-g\left(X_{(i-1) / n}\right) Y_{s-}^{n, i}\right) \mathrm{d} Y_{s}, \\
\xi_{i}^{n}(2) & =u_{n} g\left(X_{(i-1) / n}\right)\left(\int_{I(n, i)} Y_{s-}^{n, i} \mathrm{~d} \widetilde{M}_{s}^{v_{n}}+\int_{I(n, i)} \widetilde{M}_{s-}^{n, i} \mathrm{~d} \widetilde{A}_{s}^{v_{n}}\right), \\
\xi_{i}^{n}(3) & =u_{n} \int_{I(n, i)}\left(\widetilde{N}_{s-}^{\prime, i}-V_{s-}^{n}(i)\right) \mathrm{d} Y_{s}^{v_{n}}, \\
\xi_{i}^{n}(4) & =u_{n} \int_{\left[T(n, i)_{3}, i / n\right]} \widetilde{N}_{s-}^{n, i} \mathrm{~d} \widetilde{N}_{s}^{v_{n}} \mathbf{1}_{\{K(n, i) \geq 3\},}
\end{aligned}
$$




$$
\begin{aligned}
\xi_{i}^{n}(5) & =u_{n}\left(g\left(X_{T(n, i)_{1}-}\right)-g\left(X_{(i-1) / n}\right)\right) \Delta Y_{T(n, i)_{1}} \Delta Y_{T(n, i)_{2}} \mathbf{1}_{\{K(n, i) \geq 2\}}, \\
\xi_{i}^{n}(6) & =u_{n} k\left(X_{T(n, i)_{1-}}, \Delta Y_{T(n, i)_{1}}\right) \Delta Y_{T(n, i)_{1}}^{2} \Delta Y_{T(n, i)_{2}} \mathbf{1}_{\{K(n, i) \geq 2\}}, \\
\xi_{i}^{n}(7) & =u_{n} g\left(X_{(i-1) / n}\right)\left(\int_{I(n, i)} Y_{s-}^{n, i} \mathrm{~d} N_{s}^{v_{n}}-\Delta Y_{T(n, i)_{1}} Y_{T(n, i)_{1}} \mathbf{1}_{\{K(n, i) \geq 1\}}\right), \\
\xi_{i}^{n}(8) & =u_{n} k\left(X_{(i-1) / n}, \Delta Y_{T(n, i)_{1}}\right)\left(\Delta Y_{T(n, i)_{1}}\right)^{2}\left(\widetilde{M}_{i / n}^{n, i}-\widetilde{M}_{T(n, i)_{1}}^{n, i}\right), \\
\xi_{i}^{n}(9) & =u_{n} k\left(X_{(i-1) / n}, \Delta Y_{T(n, i)_{1}}\right)\left(\Delta Y_{T(n, i)_{1}}\right)^{2}\left(\widetilde{A}_{i / n}-\widetilde{A}_{T(n, i)_{1}}\right) \mathbf{1}_{\{K(n, i) \geq 1\}}, \\
\xi_{i}^{n}(10) & =u_{n} g\left(X_{(i-1) / n}\right) \Delta Y_{T(n, i)_{1}} \widetilde{M}_{i / n}^{n, i} \mathbf{1}_{\{K(n, i) \geq 1\}}, \\
\xi_{i}^{n}(11) & =u_{n} g\left(X_{(i-1) / n}\right) \int_{I(n, i)}\left(\widetilde{A}_{s-}^{n, i}+\Delta Y_{T(n, i)_{1}} \mathbf{1}_{\{K(n, i) \geq 1\}}\right) \mathrm{d} \widetilde{A}_{s}^{v_{n},}, \\
\xi_{i}^{n}(12) & =u_{n} g\left(X_{(i-1) / n}\right) \Delta Y_{T(n, i)_{1}} \Delta Y_{T(n, i)_{2}} \mathbf{1}_{\{K(n, i) \geq 2\}},
\end{aligned}
$$

where $\widetilde{\xi}_{i}^{n}(j)$ is defined in the same way, upon substituting $Y$ with $Z$.

Lemma 5. Assuming that Case I, II, or III applies, then we have $\Delta^{n}(j)_{t} \stackrel{\text { u.c.p. }}{\longrightarrow} 0$ if $j=$ $1,2, \ldots, 9$.

Proof. In the $j=1$ case, since

$$
\begin{aligned}
H_{s-}^{n, i}-g\left(X_{(i-1) / n}\right) Y_{s-}^{n, i}= & \int_{(i-1) / n}^{s} g\left(X_{r-}\right)-g\left(X_{(i-1) / n}\right)\left(\mathrm{d} \widetilde{A}_{r}^{v_{n}}+\mathrm{d} \widetilde{M}_{r}^{v_{n}}\right) \\
& +\int_{(i-1) / n}^{s} \int_{\mathbb{R}} k\left(X_{t-}, \sigma_{r-x} x\right)\left(\sigma_{r-x} x\right)^{2} \mathbf{1}_{\left\{x \leq v_{n}\right\}} \mu^{Z}(\mathrm{~d} r, \mathrm{~d} x)
\end{aligned}
$$

and $g$ is bounded with a bounded derivative, $k$ is bounded over $\mathbb{R} \times[-a, a]$.

Firstly,

$$
\begin{aligned}
& \mathbb{E}\left(\sup _{s \in I(n, i)}\left(\int_{(i-1) / n}^{s} g\left(X_{r-}\right)-g\left(X_{(i-1) / n}\right) \sigma_{r-} \mathrm{d} A_{r}^{v_{n}}\right)^{2} \mid \mathcal{F}_{(i-1) / n}\right) \\
& \quad \leq \frac{K}{n^{2}} \mathbb{E}\left(\sup _{s \in I(n, i)}\left(g\left(X_{s-}\right)-g\left(X_{(i-1) / n}\right)\right)^{2} \mid \mathcal{F}_{(i-1) / n}\right) \\
& \quad \leq \frac{K}{n^{3}} \mathbb{E}\left(\sup _{s \in I(n, i)}\left(\int_{(i-1) / n}^{s} g\left(X_{r-}\right)-g\left(X_{(i-1) / n}\right) \sigma_{r-} \mathrm{d} M_{r}^{v_{n}}\right)^{2} \mid \mathcal{F}_{(i-1) / n}\right) \\
& \quad \leq \frac{8 v_{n}^{2-\alpha}}{n} \mathbb{E}\left(\sup _{s \in I(n, i)}\left(g\left(X_{s-}\right)-g\left(X_{(i-1) / n}\right)\right)^{2} \mathcal{F}_{(i-1) / n}\right) \\
& \quad \leq \frac{8 v_{n}^{2-\alpha}}{n^{2}} \mathbb{E}\left(\operatorname { s u p } _ { s \in I ( n , i ) } \left(\int_{(i-1) / n}^{s}\right.\right. \\
& \quad \leq \frac{v_{n}^{4-\alpha}}{n}+\frac{v_{n}^{4-\alpha}}{n^{2}} .
\end{aligned}
$$


For the $j=2$ case, we firstly consider

$$
\begin{aligned}
& \mathbb{E}\left(\int_{I(n, i)} Y_{s-}^{n, i} \mathrm{~d} \tilde{M}_{s}^{v_{n}} \mid \mathcal{F}_{(i-1) / n}\right)=0 \\
& \mathbb{E}\left(\left(\int_{I(n, i)} Y_{s-}^{n, i} \mathrm{~d} \tilde{M}_{s}^{v_{n}}\right)^{2} \mid \mathcal{F}_{(i-1) / n}\right) \leq K v_{n}^{2-\alpha} \mathbb{E}\left(\int_{I(n, i)}\left(Y_{s-}^{n, i} \sigma_{s-}\right)^{2} \mathrm{~d} s \mid \mathcal{F}_{(i-1) / n}\right) \\
& \leq K \frac{v_{n}^{2-\alpha}}{n} \mathbb{E}\left(\left(\sup _{s \in I(n, i)}\left|Y_{s-}^{n, i}\right|\right)^{2} \mid \mathcal{F}_{(i-1) / n}\right) \\
& \leq K \frac{v_{n}^{2-\alpha}}{n}\left(\frac{v_{n}^{2-\alpha}}{n}+\frac{1}{n^{2} v_{n}^{2 \alpha-2}}\right)
\end{aligned}
$$

and, finally,

$$
\mathbb{E}\left(\left(\int_{I(n, i)} \tilde{M}_{s}^{v_{n}} \mathrm{~d} \widetilde{A}_{s}^{v_{n}}\right)^{2} \mid \mathcal{F}_{(i-1) / n}\right) \leq \frac{v_{n}^{4-3 \alpha}}{n^{3}} .
$$

We obtain $\Delta^{n}(2)_{t} \stackrel{\text { u.c.p. }}{\longrightarrow} 0$.

The $j=3$ case is similar to Jacod [2]. We have ${\widetilde{N^{\prime}}}_{s-}^{n, i}-V_{s-}^{n}(i)=U^{n, i} * \mu^{Z}$, where

$$
\begin{aligned}
U^{n, i}(s, & x) \\
= & \left(g\left(X_{s-}\right) \mathbf{1}_{\left\{s>T(n, i)_{1}\right\}}+\left(g\left(X_{s-}\right)-g\left(X_{(i-1) / n}\right)\right) \mathbf{1}_{\left\{(i-1) / n<s \leq T(n, i)_{1}\right\}}\right) \sigma_{s-} x \mathbf{1}_{\left\{|x|>v_{n}\right\}} \\
& +\left(k\left(X_{s-}, \sigma_{s-} x\right) \mathbf{1}_{\left\{s>T(n, i)_{1}\right\}}+\left(k\left(X_{s-}, \sigma_{s-} x\right)\right.\right. \\
& \left.\left.-k\left(X_{(i-1) / n}, \sigma_{s-} x\right)\right) \mathbf{1}_{\left\{(i-1) / n<s \leq T(n, i)_{1}\right\}}\right)\left(\sigma_{s-} x\right)^{2} \mathbf{1}_{\left\{|x|>v_{n}\right\}} .
\end{aligned}
$$

We have

$$
\begin{gathered}
\left|\int_{\mathbb{R}} U^{n, i}(t, x) G_{t}(\mathrm{~d} x)\right| \leq C\left(\sup _{t \in I(n, i)} d_{t}^{\prime}\left(v_{n}\right)+1\right)\left(\mathbf{1}_{\{K(n, i) \geq 1\}}+\widetilde{X}_{i}^{n}\right), \\
\int_{\mathbb{R}} U^{n, i}(t, x)^{2} G_{t}(\mathrm{~d} x) \leq C\left(\mathbf{1}_{\{K(n, i) \geq 1\}}+\left(\widetilde{X}_{i}^{n}\right)^{2}\right) .
\end{gathered}
$$

Then

$$
\begin{gathered}
\sup _{s \in I(n, i)}\left|\mathbb{E}\left(\widetilde{N}_{s-}^{n, i}-V_{s-}^{n}(i) \mid \mathcal{F}_{(i-1) / n}\right)\right| \leq C \frac{1}{n}\left(1+\sup _{t \in I(n, i)} d_{t}^{\prime}\left(v_{n}\right)\right)\left(\frac{1}{n v_{n}^{\alpha}}+\frac{1}{\sqrt{n}}\right), \\
\mathbb{E}\left(\sup _{s \in I(n, i)}\left|\widetilde{N}_{s-}^{n, i}-V_{s-}^{n}(i)\right|^{2} \mid \mathcal{F}_{(i-1) / n}\right) \leq C \frac{1}{n}\left(1+\frac{\left(\sup _{t \in I(n, i)} d_{t}^{\prime}\left(v_{n}\right)\right)^{2}}{n}\right)\left(\frac{1}{n v_{n}^{\alpha}}+\frac{1}{n}\right) .
\end{gathered}
$$

The $j=4$ case is similar to Jacod [2]. Set $J_{r}=\left(\Delta H_{T(n, i)_{1}}+\Delta H_{T(n, i)_{2}}+\cdots+\right.$ $\left.\Delta H_{T(n, i)_{r-1}}\right) \sigma_{T(n, i)_{r-}}, J_{r}^{\prime}=J_{r} \Delta Z_{T(n, i)_{r}}$, and $J_{k}^{\prime \prime}=u_{n} \sum_{r=3}^{k} J_{r}^{\prime}$. Then

$$
\xi_{i}^{n}(4)=J_{K(n, i)}^{\prime \prime} \mathbf{1}_{\{K(n, i) \geq 3\}} \text {. }
$$

We need to prove that

$$
\begin{gathered}
\mathbb{P}\left(\left|\xi_{i}^{n}(4)\right|>y \mid \mathcal{F}_{(i-1) / n}\right) \leq \frac{a_{n, y}^{\prime \prime}}{n}, \quad \text { where } a_{n, y}^{\prime \prime} \rightarrow 0 ; \\
\mathbb{E}\left(\left|\xi_{i}^{n}(4)\right|^{2} \mathbf{1}_{\left\{\left|\tilde{\xi}_{i}^{n}(4)\right| \leq 1\right\}} \mid \mathcal{F}_{(i-1) / n}\right) \leq \frac{a_{n}^{\prime}}{n}, \quad \text { where } a_{n}^{\prime} \rightarrow 0 ; \\
\left|\mathbb{E}\left(\xi_{i}^{n}(4) \mathbf{1}_{\left\{\left|\tilde{\xi}_{i}^{n}(4)\right| \leq 1\right\}} \mid \mathcal{F}_{(i-1) / n}\right)\right| \leq \frac{a_{n}}{n}, \quad \text { where } a_{n} \rightarrow 0 .
\end{gathered}
$$


In view of (18), for $y>0, k \geq 3$, we have

$$
\begin{aligned}
\mathbb{P}\left(\left|\xi_{i}^{n}(4)\right|>y \mid \mathcal{F}_{(i-1) / n}\right) & =\sum_{k=3}^{\infty} \mathbb{P}\left(\left|J_{k}^{\prime \prime}\right|>y, K(n, i)=k \mid \mathcal{F}_{(i-1) / n}\right) \\
& \leq \sum_{k=3}^{\infty} \sum_{r=3}^{k} \mathbb{P}\left(\left|u_{n} J_{r}^{\prime}\right|>\frac{y}{k-2}, K(n, i)=k \mid \mathcal{F}_{(i-1) / n}\right) \\
& \leq C \sum_{k=3}^{\infty} \sum_{r=3}^{k} \frac{(r-1)^{\alpha+1} u_{n}^{\alpha}(k-2)^{\alpha} \log \left(1 / v_{n}\right)}{\left(v_{n}\right)^{\alpha(k-2)} n^{k} y^{\alpha}} \\
& =o\left(\frac{1}{n}\right) .
\end{aligned}
$$

In view of (19), for $k \geq 3$, we have

$$
\begin{aligned}
\mathbb{E}\left(\left|\xi_{i}^{n}(4)\right|^{2} \mathbf{1}_{\left\{\left|\xi_{i}^{n}(4)\right| \leq 1\right\}} \mid \mathcal{F}_{(i-1) / n}\right) \\
=\sum_{k=3}^{\infty} \mathbb{E}\left(\left|J_{k}^{\prime \prime}\right|^{2} \mathbf{1}_{\left\{\left|J_{k}^{\prime \prime}\right| \leq 1, K(n, i)=k\right\}} \mid \mathcal{F}_{(i-1) / n}\right) \\
\leq \sum_{k=3}^{\infty} \sum_{r=3}^{k-1} 2^{k-r} \mathbb{P}\left(\left|J_{r}^{\prime \prime}\right|>1, K(n, i)=k \mid \mathcal{F}_{(i-1) / n}\right) \\
\quad+\sum_{k=3}^{\infty} \sum_{r=3}^{k} 2^{k+1-r} \mathbb{E}\left(\left(u_{n} J_{r}^{\prime}\right)^{2} \mathbf{1}_{\left\{\left|u_{n} J_{r}^{\prime}\right| \leq 2, K(n, i)=k\right\}} \mid \mathcal{F}_{(i-1) / n}\right) \\
\leq C \sum_{k=3}^{\infty}\left(\frac{2^{k} k^{2 \alpha+3} u_{n}^{\alpha} \log \left(1 / v_{n}\right)}{n^{k}\left(v_{n}\right)^{\alpha(k-2)}}+\frac{2^{k} k^{4} u_{n}^{\alpha} \log \left(1 / v_{n}\right)}{n^{k}\left(v_{n}\right)^{\alpha(k-2)}}\right) \\
=o\left(\frac{1}{n}\right) .
\end{aligned}
$$

We have

$$
\left|\mathbb{E}\left(\xi_{i}^{n}(4) \mathbf{1}_{\left\{\left|\xi_{i}^{n}(4)\right| \leq 1\right\}} \mid \mathcal{F}_{(i-1) / n}\right)\right|=\sum_{k=3}^{\infty}\left|\mathbb{E}\left(J_{k}^{\prime \prime} \mathbf{1}_{\left\{\left|J_{k}^{\prime \prime}\right| \leq 1, K(n, i)=k\right\}} \mid \mathcal{F}_{(i-1) / n}\right)\right|,
$$

and

$$
\begin{aligned}
&\left|\mathbb{E}\left(J_{k}^{\prime \prime} \mathbf{1}_{\left\{\left|J_{k}^{\prime \prime}\right| \leq 1, K(n, i)=k\right\}} \mid \mathcal{F}_{(i-1) / n}\right)\right| \\
& \leq\left|\mathbb{E}\left(J_{k-1}^{\prime \prime} \mathbf{1}_{\left\{\left|J_{k-1}^{\prime \prime}\right| \leq 1 / 2, K(n, i)=k\right\}} \mid \mathcal{F}_{(i-1) / n}\right)\right| \\
&+\left|\mathbb{E}\left(u_{n} J_{k}^{\prime} \mathbf{1}_{\left\{\left|u_{n} J_{k}^{\prime}\right| \leq 1 / 2,\left|J_{k}^{\prime \prime}\right| \leq 1, K(n, i)=k\right\}} \mid \mathcal{F}_{(i-1) / n}\right)\right| \\
&+\mathbb{E}\left(\left|J_{k}^{\prime \prime} \mathbf{1}_{\left\{\left|J_{k}^{\prime \prime}\right| \leq 1,\left|J_{k-1}^{\prime \prime}\right| \geq 1 / 2\right\}}-J_{k-1}^{\prime \prime} \mathbf{1}_{\left\{\left|J_{k-1}^{\prime \prime}\right| \leq 1 / 2,\left|J_{k}^{\prime \prime}\right| \geq 1\right\}}\right| \mathbf{1}_{\{K(n, i)=k\}} \mid \mathcal{F}_{(i-1) / n}\right) .
\end{aligned}
$$

When $\alpha \geq 1$, by (16), we have

$$
\begin{aligned}
\left|\mathbb{E}\left(u_{n} J_{k}^{\prime} \mathbf{1}_{\left\{\left|u_{n} J_{k}^{\prime}\right| \leq 1 / 2,\left|J_{k}^{\prime \prime}\right| \leq 1, K(n, i)=k\right\}} \mid \mathcal{F}_{(i-1) / n}\right)\right| & \leq \mathbb{E}\left(\left|u_{n} J_{k}^{\prime}\right| \mathbf{1}_{\{K(n, i)=k\}} \mid \mathcal{F}_{(i-1) / n}\right) \\
& \leq K k \frac{(\log n)^{2}}{\left(v_{n}^{\alpha}\right)^{k-1} n^{k}} .
\end{aligned}
$$


When $\alpha<1$, by (17), we have

$$
\begin{aligned}
& \left|\mathbb{E}\left(u_{n} J_{k}^{\prime} \mathbf{1}_{\left\{\left|u_{n} J_{k}^{\prime}\right| \leq 1 / 2,\left|J_{k}^{\prime \prime}\right| \leq 1, K(n, i)=k\right\}} \mid \mathcal{F}_{(i-1) / n}\right)\right| \\
& \leq \mathbb{E}\left(\left|u_{n} J_{k}^{\prime}\right| \mathbf{1}_{\left\{\left|u_{n} J_{k}^{\prime}\right| \leq 1 / 2, K(n, i)=k\right\}} \mid \mathcal{F}_{(i-1) / n}\right) \\
& \quad \leq \frac{K u_{n}^{\alpha} k^{2}\left(1+\log \left(1 / v_{n}\right)\right)^{2}}{\left(v_{n}^{\alpha}\right)^{k-2} n^{k}} .
\end{aligned}
$$

Obviously,

$$
\begin{aligned}
\mathbb{E}\left(\left|J_{k}^{\prime \prime} \mathbf{1}_{\left\{\left|J_{k}^{\prime \prime}\right| \leq 1,\left|J_{k-1}^{\prime \prime}\right| \geq 1 / 2\right\}}-J_{k-1}^{\prime \prime} \mathbf{1}_{\left\{\left|J_{k-1}^{\prime \prime}\right| \leq 1 / 2,\left|J_{k}^{\prime \prime}\right| \geq 1\right\}}\right| \mathbf{1}_{\{K(n, i)=k\}} \mid \mathcal{F}_{(i-1) / n}\right) \\
\leq \sum_{r=3}^{k} \frac{(r-1)^{\alpha+1} u_{n}^{\alpha}(k-2)^{\alpha} \log \left(1 / v_{n}\right)}{\left(v_{n}\right)^{\alpha(k-2)} n^{k}} \\
\leq \frac{(k-1)^{2 \alpha+2} u_{n}^{\alpha} \log \left(1 / v_{n}\right)}{\left(v_{n}\right)^{\alpha(k-2)} n^{k}}
\end{aligned}
$$

Furthermore,

$$
\begin{aligned}
\left|\mathbb{E}\left(J_{k}^{\prime \prime} \mathbf{1}_{\left\{\left|J_{k}^{\prime \prime}\right| \leq 1, K(n, i)=k\right\}} \mid \mathcal{F}_{(i-1) / n}\right)\right| \leq & \left|\mathbb{E}\left(J_{k-1}^{\prime \prime} \mathbf{1}_{\left\{\left|J_{k-1}^{\prime \prime}\right| \leq 1 / 2, K(n, i)=k\right\} \mid} \mid \mathcal{F}_{(i-1) / n}\right)\right| \\
& +K \frac{(k)^{2 \alpha+2} u_{n}^{\alpha}\left(\log \left(1 / v_{n}\right)+(\log n)^{2}\right)}{\left(v_{n}\right)^{\alpha(k-2)} n^{k}} .
\end{aligned}
$$

Recalling that $J_{2}^{\prime \prime}=0$, then

$$
\left|\mathbb{E}\left(J_{k}^{\prime \prime} \mathbf{1}_{\left\{\left|J_{k}^{\prime \prime}\right| \leq 1, K(n, i)=k\right\}} \mid \mathcal{F}_{(i-1) / n}\right)\right| \leq K \frac{k^{2 \alpha+3} u_{n}^{\alpha}\left(\log \left(1 / v_{n}\right)+(\log n)^{2}\right)}{\left(v_{n}\right)^{\alpha(k-2)} n^{k}}
$$

and

$$
\begin{aligned}
\left|\mathbb{E}\left(J_{k}^{\prime \prime} \mathbf{1}_{\left\{\left|J_{k}^{\prime \prime}\right| \leq 1, K(n, i) \geq 3\right\}} \mid \mathcal{F}_{(i-1) / n}\right)\right| & \leq K \sum_{k=3}^{\infty} \frac{k^{2 \alpha+3} u_{n}^{\alpha}\left(\log \left(1 / v_{n}\right)+(\log n)^{2}\right)}{\left(v_{n}\right)^{\alpha(k-2)} n^{k}} \\
& \leq \frac{K u_{n}^{\alpha}\left(\log \left(1 / v_{n}\right)+(\log n)^{2}\right)}{n^{2}} .
\end{aligned}
$$

In the $j=5$ case,

$$
\left|\xi_{i}^{n}(5)\right| \leq u_{n} \widetilde{Z}_{T(n, i)_{1}}^{\prime n}\left|\Delta Y_{T(n, i)_{1}} \Delta Y_{T(n, i)_{2}}\right| \mathbf{1}_{\{K(n, i) \geq 2\}},
$$

where $\widetilde{Z}_{T(n, i)_{1}}^{\prime n}=\sup _{s \in\left[(i-1) / n, T(n, i)_{1}\right]}\left|Z_{s-}^{v_{n}}-Z_{(i-1) / n}^{v_{n}}\right|$.

Since $\sigma$ is bounded, we need to prove that

$$
u_{n} \widetilde{Z}_{T(n, i)_{1}}^{\prime n}\left|\Delta Z_{T(n, i)_{1}} \Delta Z_{T(n, i)_{2}}\right| \mathbf{1}_{\{K(n, i) \geq 2\}} \stackrel{\text { u.c.p. }}{\longrightarrow} 0 .
$$

Since

$$
\mathbb{E}\left[\left(\widetilde{Z}_{T(n, i)_{1}}^{\prime n}\right)^{2} \mid \mathcal{F}_{(i-1) / n}\right] \leq\left(\frac{\sup _{t \in I(n, i)} c_{t}\left(v_{n}\right)}{n}+\frac{\left(\sup _{t \in I(n, i)} d_{t}\left(v_{n}\right)\right)^{2}}{n^{2}}\right)
$$


and using Equation (6.35) of [1], we have, when $0<\alpha<1$,

$$
\begin{aligned}
\mathbb{E}\left[\mid u_{n}\right. & \left.\tilde{Z}_{T(n, i)_{1}}^{\prime n}\left|\Delta Z_{T(n, i)_{1}} \Delta Z_{T(n, i)_{2}}\right| \mathbf{1}_{\{K(n, i) \geq 2\}}|| \mathcal{F}_{(i-1) / n}\right] \\
& \leq u_{n} \frac{\left(\sup _{t \in I(n, i)} c_{t}\left(v_{n}\right) / n+\left(\sup _{t \in I(n, i)} d_{t}\left(v_{n}\right)\right)^{2} / n^{2}\right)^{1 / 2}}{n^{2}} \\
& =o\left(\frac{1}{n}\right),
\end{aligned}
$$

when $\alpha=1$,

$$
\begin{aligned}
\mathbb{E}\left[\mid u_{n}\right. & \left.\widetilde{Z}_{T(n, i)_{1}}^{\prime n}\left|\Delta Z_{T(n, i)_{1}} \Delta Z_{T(n, i)_{2}}\right| \mathbf{1}_{\{K(n, i) \geq 2\}}|| \mathcal{F}_{(i-1) / n}\right] \\
& \leq u_{n} \frac{\left(\sup _{t \in I(n, i)} c_{t}\left(v_{n}\right) / n+\left(\sup _{t \in I(n, i)} d_{t}\left(v_{n}\right)\right)^{2} / n^{2}\right)^{1 / 2}(\log n)^{2}}{n^{2}} \\
& =o\left(\frac{1}{n}\right),
\end{aligned}
$$

when $\alpha>1$,

$$
\begin{aligned}
\mathbb{E}\left[\mid u_{n}\right. & \left.\widetilde{Z}_{T(n, i)_{1}}^{\prime n}\left|\Delta Z_{T(n, i)_{1}} \Delta Z_{T(n, i)_{2}}\right| \mathbf{1}_{\{K(n, i) \geq 2\}}|| \mathcal{F}_{(i-1) / n}\right] \\
& \leq u_{n} \frac{\left(\sup _{t \in I(n, i)} c_{t}\left(v_{n}\right) / n+\left(\sup _{t \in I(n, i)} d_{t}\left(v_{n}\right)\right)^{2} / n^{2}\right)^{1 / 2}}{\left(v_{n}^{\alpha-1} n\right)^{2}} \\
& =o\left(\frac{1}{n}\right),
\end{aligned}
$$

and

$$
\begin{aligned}
\mathbb{E}\left[\mid u_{n}\right. & \left.\left.\widetilde{Z}_{T(n, i)_{1}}^{\prime n}\left|\Delta Z_{T(n, i)_{1}} \Delta Z_{T(n, i)_{2}}\right| \mathbf{1}_{\{K(n, i) \geq 2\}}\right|^{2} \mid \mathcal{F}_{(i-1) / n}\right] \\
& \leq u_{n}^{2} \frac{\left(\sup _{t \in I(n, i)} c_{t}\left(v_{n}\right) / n+\left(\sup _{t \in I(n, i)} d_{t}\left(v_{n}\right)\right)^{2} / n^{2}\right)}{n^{2}} \\
& =o\left(\frac{1}{n}\right) .
\end{aligned}
$$

By Lemma 4, we obtain (20).

In the $j=6$ case, set $S_{i}^{n}=u_{n} \Delta Z_{T(n, i)_{1}}^{2}\left|\Delta Z_{T(n, i)_{2}}\right| \mathbf{1}_{\{K(n, i) \geq 2\}}$, we have

$$
\begin{aligned}
& \mathbb{P}\left(S_{i}^{n}>y \mid \mathcal{F}_{(i-1) / n}\right) \\
& \quad \leq \int_{\left\{s_{0}<s_{1}<s_{2}<i / n\right\}} \prod_{l=1}^{2} \alpha_{s_{l-1}, s_{l}}^{n} \bar{G}_{s_{l}}\left(v_{n}\right) \mathbb{P}\left(u_{n} \Delta Z_{T(n, i) 1}^{2}\left|\Delta Z_{T(n, i)_{2}}\right|>y \mid \mathcal{F}_{(i-1) / n}\right) \mathrm{d} s_{1} \mathrm{~d} s_{2} \\
& \quad \leq \int_{\left\{s_{0}<s_{1}<s_{2}<i / n\right\}} \prod_{l=1}^{2} \alpha_{s_{l-1}, s_{l}}^{n} \int_{\left\{|x|>v_{n}\right\}} \int_{\left\{\left|x^{\prime}\right|>y / u_{n} x^{2}\right\}} G_{s_{1}}(\mathrm{~d} x) G_{s_{2}}\left(\mathrm{~d} x^{\prime}\right) \mathrm{d} s_{1} \mathrm{~d} s_{2} \\
& \quad \leq \int_{\left\{s_{0}<s_{1}<s_{2}<i / n\right\}} \prod_{l=1}^{2} \alpha_{s_{l-1}, s_{l}}^{n} \int \frac{u_{n}^{\alpha}|x|^{2 \alpha}}{y^{\alpha}} G_{s_{1}}(\mathrm{~d} x) \mathrm{d} s_{1} \mathrm{~d} s_{2} \\
& \quad<C \frac{u_{n}^{\alpha}}{n^{2} y^{\alpha}}
\end{aligned}
$$


and

$$
\begin{aligned}
& \mathbb{E}\left(S_{i}^{n} \mathbf{1}_{\left\{S_{i}^{n} \leq 1\right\}} \mid \mathcal{F}_{(i-1) / n}\right) \\
& \leq \int_{\left\{s_{0}<s_{1}<s_{2}<i / n\right\}} \prod_{l=1}^{2} \alpha_{s_{l-1}, s_{l}}^{n} \bar{G}_{s_{l}}\left(v_{n}\right) \\
& \quad \times \mathbb{E}\left(u_{n} \Delta Z_{T(n, i) 1}^{2}\left|\Delta Z_{T(n, i)_{2}}\right| \mathbf{1}_{\left\{u_{n} \Delta Z_{T(n, i)}^{2}\left|\Delta Z_{T(n, i)}\right| \leq 1\right\}} \mid \mathcal{F}_{(i-1) / n}\right) \mathrm{d} s_{1} \mathrm{~d} s_{2} \\
& \leq \\
& \leq \int_{\left\{s_{0}<s_{1}<s_{2}<i / n\right\}} \prod_{l=1}^{2} \alpha_{s_{l-1}, s_{l}}^{n} \int_{\left\{\left|x^{\prime}\right|>v_{n}\right\}}\left|x^{\prime}\right| \int_{\left\{|x| \leq 1 / \sqrt{\left.u_{n}\left|x^{\prime}\right|\right\}}\right.} x^{2} G_{s_{1}}(\mathrm{~d} x) G_{s_{2}}\left(\mathrm{~d} x^{\prime}\right) \mathrm{d} s_{1} \mathrm{~d} s_{2} \\
& \leq \int_{\left\{s_{0}<s_{1}<s_{2}<i / n\right\}} \prod_{l=1}^{2} \alpha_{s_{l-1}, s_{l}}^{n} \int_{\left\{\left|x^{\prime}\right|>v_{n}\right\}} u_{n}^{\alpha / 2}\left|x^{\prime}\right|^{\alpha / 2} G_{s_{2}}\left(\mathrm{~d} x^{\prime}\right) \mathrm{d} s_{1} \mathrm{~d} s_{2} \\
&< C \frac{u_{n}^{\alpha / 2}}{n^{2} v_{n}^{\alpha / 2}} .
\end{aligned}
$$

Since $k(x, y)$ and $\sigma$ are bounded, we have $\left|\xi_{i}^{n}(6)\right| \leq C S_{i}^{n}$, and if $\left|S_{i}^{n}\right| \leq 1$,

$$
\left(S_{i}^{n}\right)^{2} \leq\left|S_{i}^{n}\right| \leq S_{i}^{n} \mathbf{1}_{\left\{S_{i}^{n} \leq 1\right\}}+1_{\left\{S_{i}^{n}>1\right\}}
$$

Then

$$
\Delta^{n}(6)_{t} \stackrel{\text { u.c.p. }}{\longrightarrow} 0
$$

by Lemma 4 .

In the $j=7$ case, it is obvious that

$$
\left|\xi_{i}^{n}(7)\right| \leq C \sum_{k=2}^{K} H_{k}, \quad \text { where } H_{k}=u_{n} Z_{T(n, k) \wedge i / n}^{n, i} \Delta Z_{T(n, k)} .
$$

We have

$$
\begin{aligned}
\mathbb{E}\left[\left(\sum_{k=2}^{K} H_{k}\right)^{2} \mid \mathcal{F}_{(i-1) / n}\right] \leq & u_{n}^{2} \mathbb{E}\left[K \sum_{k=2}^{K}\left(Z_{T(n, k) \wedge i / n}^{n, i} \Delta Z_{T(n, k)}\right)^{2} \mid \mathcal{F}_{(i-1) / n}\right] \\
\leq & u_{n}^{2} \vartheta_{n} \sum_{r=2}^{\infty} r \sum_{k=2}^{r} \int_{\left\{s_{0}<s_{1}<\cdots<s_{r}<i / n\right\}} \prod_{l=1}^{r} \alpha_{s_{l-1}, s_{l}}^{n} \\
& \times \prod_{l=1, l \neq k}^{r} \bar{G}_{s_{l}}\left(v_{n}\right) \int_{\left\{|x| \geq v_{n}\right\}}|x|^{2} G_{s_{k}}(\mathrm{~d} x) \mathrm{d} s_{1} \mathrm{~d} s_{2} \cdots \mathrm{d} s_{r} \\
\leq & u_{n}^{2} \vartheta_{n} \sum_{r=2}^{\infty} \frac{C r(r-1)}{\left(v_{n}\right)^{\alpha-1} n^{r}} \\
= & o\left(\frac{1}{n}\right),
\end{aligned}
$$

where

$$
\vartheta_{n}=\frac{\sup _{t \in I(n, i)} c_{t}\left(v_{n}\right)}{n}+\frac{\left(\sup _{t \in I(n, i)} d_{t}\left(v_{n}\right)\right)^{2}}{n^{2}}
$$


and

$$
\begin{aligned}
\left|\mathbb{E}\left[\sum_{k=2}^{K} H_{k} \mid \mathcal{F}_{(i-1) / n}\right]\right| & \frac{C u_{n}\left|\sup _{t \in I(n, i)} d_{t}\left(v_{n}\right)\right|}{n} \mathbb{E}\left[\sum_{k=2}^{K}\left|\Delta Z_{T(n, k)}\right| \mid \mathcal{F}_{(i-1) / n}\right] \\
\leq & \frac{C u_{n}\left|\sup _{t \in I(n, i)} d_{t}\left(v_{n}\right)\right|}{n} \sum_{r=2}^{\infty} \sum_{k=2}^{r} \int_{\left\{s_{0}<s_{1}<\cdots<s_{r}<i / n\right\}} \prod_{l=1}^{r} \alpha_{s_{l-1}, s_{l}}^{n} \\
& \times \prod_{l=1, l \neq k}^{r} \bar{G}_{s_{l}}\left(v_{n}\right) \int_{\left\{|x| \geq v_{n}\right\}}|x| G_{s_{k}}(\mathrm{~d} x) \mathrm{d} s_{1} \mathrm{~d} s_{2} \cdots \mathrm{d} s_{r} \\
\leq & \frac{C u_{n}\left|\sup _{t \in I(n, i)} d_{t}\left(v_{n}\right)\right|}{n} \sum_{r=2}^{\infty} \frac{C(r-1) \sup _{t \in I(n, i)} \delta_{t}\left(v_{n}\right)}{\left(v_{n}\right)^{\alpha-1} n^{r}} \\
= & o\left(\frac{1}{n}\right) .
\end{aligned}
$$

Then

$$
\Delta^{n}(7)_{t} \stackrel{\text { u.c.p. }}{\longrightarrow} 0
$$

by Lemma 4 .

In the $j=8$ case, from the previous discussion, it suffices to prove that

$$
\sum_{i=1}^{[n t]} \zeta_{i}^{n}:=\sum_{i=1}^{[n t]} u_{n}\left(\Delta Z_{T(n, i)_{1}}\right)^{2}\left(M_{i / n}^{n, i}-M_{T(n, i)_{1}}^{n, i}\right) \stackrel{\text { u.c.p. }}{\longrightarrow} 0 .
$$

Set

$$
\begin{gathered}
\phi_{n, i}(u)=\mathbb{E}\left[\exp \left(i u \zeta_{i}^{n}\right)\right], \\
z_{i}^{n}(x, u, t)=\int_{t}^{i / n} \mathrm{~d} s \int_{\left\{|y| \leq v_{n}\right\}}\left(\exp \left(i u u_{n} x^{2} y\right)-1-i u u_{n} x^{2} y\right) G_{s}(y),
\end{gathered}
$$

we see that

$$
\phi_{n, i}(u)=\alpha_{(i-1) / n, i / n}^{n} \int_{I(n, i)} \mathrm{d} t \int_{\left\{|x|>v_{n} \mid\right\}}\left(\exp \left(z_{i}^{n}(x, u, t)-1\right)\right) G_{t}(\mathrm{~d} x)+1 .
$$

In fact,

$$
\begin{aligned}
& \int_{t}^{i / n} \mathrm{~d} s \int_{\left\{|y| \leq v_{n}\right\}}\left(\exp \left(i u u_{n} x^{2} y\right)-1-i u u_{n} x^{2} y\right) G_{s}(y) \\
& \leq \int_{t}^{i / n} \mathrm{~d} s \int_{\left\{|y| \leq v_{n}\right\}}\left(\left|u u_{n} x^{2} y\right| \wedge\left|u u_{n} x^{2} y\right|^{2}\right) G_{s}(y) \\
& \leq u u_{n} x^{2} \int_{t}^{i / n} \mathrm{~d} s \int_{\left\{|y|>1 / u u_{n} x^{2}\right\}}(|y|) G_{s}(y) \\
& \quad+\left(u u_{n} x^{2}\right)^{2} \int_{t}^{i / n} \mathrm{~d} s \int_{\left\{|y| \leq 1 / u u_{n} x^{2}\right\}}\left(y^{2}\right) G_{S}(y) \\
& \leq \frac{C}{n}\left|u u_{n} x^{2}\right|^{\alpha} .
\end{aligned}
$$


Thus, $z_{i}^{n}$ is uniformly bounded, then

$$
\left|\phi_{n, i}(u)-1\right| \leq \frac{C\left|u u_{n}\right|^{\alpha}}{n^{2}} .
$$

Furthermore,

$$
\begin{gathered}
\mathbb{E}\left[\left|\zeta_{i}^{n}\right|^{2} \wedge 1 \mid \mathcal{F}_{(i-1) / n}\right] \leq C \int_{\{|u| \leq 1\}}\left|1-\mathbb{E}\left[\exp \left(i u \zeta_{i}^{n}\right) \mid \mathcal{F}_{(i-1) / n}\right]\right| \mathrm{d} u \leq \frac{C\left|u_{n}\right|^{\alpha}}{n^{2}}, \\
\left|\mathbb{E}\left[\zeta_{i}^{n} \mathbf{1}_{\left\{\left|\zeta_{i}^{n}\right| \leq 1\right\}} \mid \mathcal{F}_{(i-1) / n}\right]\right| \leq C\left|1-\mathbb{E}\left[\exp \left(i \zeta_{i}^{n}\right) \mid \mathcal{F}_{(i-1) / n}\right]\right|+\mathbb{E}\left[\left|\zeta_{i}^{n}\right|^{2} \wedge 1 \mid \mathcal{F}_{(i-1) / n}\right] .
\end{gathered}
$$

Then

$$
\Delta^{n}(8)_{t} \stackrel{\text { u.c.p. }}{\longrightarrow} 0
$$

by Lemma 4.

For the $j=9$ case,

$$
\left|\xi_{i}^{n}(9)\right| \leq \frac{C u_{n}\left|\sup _{t \in I(n, i)} d_{t}\left(v_{n}\right)\right|}{n}\left|\Delta Z_{T(n, i)_{1}}\right|^{2} \mathbf{1}_{\{K(n, i) \geq 1\}}
$$

and

$$
\begin{aligned}
& \mathbb{E}\left[\left|\Delta Z_{T(n, i)_{1}}\right|^{2} \mathbf{1}_{\{K(n, i) \geq 1\}} \mid \mathcal{F}_{(i-1) / n}\right] \\
& \quad \leq \int_{\left\{s_{0}<s_{1}<i / n\right\}} \alpha_{s_{0}, s_{1}}^{n} \bar{G}_{s_{l}}\left(v_{n}\right) \mathbb{E}\left(\left|\Delta Z_{T(n, i) 1}\right|^{2} \mid \mathcal{F}_{(i-1) / n}\right) \mathrm{d} s_{1} \\
& \quad \leq \int_{\left\{s_{0}<s_{1}<i / n\right\}} \alpha_{s_{0}, s_{1}}^{n} \int_{\left\{|x|>v_{n}\right\}}|x|^{2} G_{s_{1}}(\mathrm{~d} x) \mathrm{d} s_{1} \\
& \quad \leq \frac{C}{n}
\end{aligned}
$$

Thus,

$$
\mathbb{E}\left[\xi_{i}^{n}(9) \mid \mathcal{F}_{(i-1) / n}\right] \leq \frac{C u_{n}\left|\sup _{t \in I(n, i)} d_{t}\left(v_{n}\right)\right|}{n^{2}}
$$

Then

$$
\Delta^{n}(9)_{t} \stackrel{\text { u.c.p. }}{\longrightarrow} 0
$$

by Lemma 4 .

\section{The proof of Theorem 1}

To prove Theorem 1, we need to study the asymptotics of $\sum_{i=1}^{[n t]} \xi_{i}^{n}(10), \sum_{i=1}^{[n t]} \xi_{i}^{n}(11)$, and $\sum_{i=1}^{[n t]} \xi_{i}^{n}(12)$. Set

$$
\begin{aligned}
& \widetilde{\xi}_{i}^{n}(10)=u_{n} \Delta Y_{T(n, i){ }_{1}} \tilde{M}_{i / n}^{n, i} \mathbf{1}_{\{K(n, i) \geq 1\}}, \\
& \widetilde{\xi}_{i}^{n}(11)=u_{n} \int_{I(n, i)}\left(\widetilde{A}_{s-}^{n, i}+\Delta Y_{T(n, i)_{1}} \mathbf{1}_{\{K(n, i) \geq 1\}}\right) \mathrm{d} \widetilde{A}_{s}^{v_{n}}, \\
& \widetilde{\xi}_{i}^{n}(12)=u_{n} \Delta Y_{T(n, i)_{1}} \Delta Y_{T(n, i)_{2}} \mathbf{1}_{\{K(n, i) \geq 2\}} .
\end{aligned}
$$


From Proposition 7.3 and Lemma 7.5 of [1], the following arguments can be obtained.

In case I,

$$
\sum_{i=1}^{[n t]} \widetilde{\xi}_{i}^{n}(11) \stackrel{\text { u.c.p. }}{\longrightarrow} 0, \quad \sum_{i=1}^{[n t]} \widetilde{\xi}_{i}^{n}(12) \stackrel{\text { u.c.p. }}{\longrightarrow} 0, \quad \sum_{i=1}^{[n \cdot]} \widetilde{\xi}_{i}^{n}(10) \Longrightarrow V
$$

where $V$ is another Lévy process, independent of $Z$, with spot characteristics $\left(b_{t}^{V}, 0, G_{t}^{V}\right)$ given by

$$
\begin{gathered}
b_{t}^{V}=\frac{-\alpha\left(\theta_{t}^{\prime}\right)^{2}}{2(\alpha-1)}, \\
G_{t}^{V}(\mathrm{~d} x)=\frac{\alpha}{2}\left[\left(\left(\theta_{t}^{+}\right)^{2}+\left(\theta_{t}^{-}\right)^{2}\right) \mathbf{1}_{\{x>0\}}+2 \theta_{t}^{+} \theta_{t}^{-} \mathbf{1}_{\{x<0\}}\right] \frac{1}{|x|^{1+\alpha}} \mathrm{d} x .
\end{gathered}
$$

In case II,

$$
\sum_{i=1}^{[n t]} \widetilde{\xi}_{i}^{n}(10) \stackrel{\text { u.c.p. }}{\longrightarrow} 0, \quad \sum_{i=1}^{[n \cdot]} \widetilde{\xi}_{i}^{n}(11)+\sum_{i=1}^{[n \cdot]} \widetilde{\xi}_{i}^{n}(12) \stackrel{\text { u.c.p. }}{\longrightarrow}-\frac{1}{4} \int_{0}^{\cdot} \theta_{s}^{\prime 2} \mathrm{~d} s ;
$$

In case III,

$$
\sum_{i=1}^{[n t]} \widetilde{\xi}_{i}^{n}(10) \stackrel{\text { u.c.p. }}{\longrightarrow} 0, \quad \sum_{i=1}^{[n t]} \widetilde{\xi}_{i}^{n}(11) \stackrel{\text { u.c.p. }}{\longrightarrow} 0, \quad \sum_{i=1}^{[n \cdot]} \widetilde{\xi}_{i}^{n}(12) \quad \Longrightarrow V
$$

where $V$ is another Lévy process, independent of $Z$, with spot characteristics $\left(b_{t}^{V}, 0, G_{t}^{V}\right)$ given by

$$
b_{t}^{V}=0, \quad G_{t}^{V}(\mathrm{~d} x)=\frac{\theta_{t}^{2} \alpha}{4|x|^{1+\alpha}} \mathrm{d} x .
$$

Finally, we obtain the results by means of Theorem 3.2 of [3].

\section{Acknowledgements}

The author would like to thank the Editor and anonymous referee for their valuable suggestions which have greatly improved this paper. The author is supported by a General Financial Grant from the China Postdoctoral Science Foundation (number 2011M500985) and Hong Kong Scholars Program (number XJ2013001).

\section{References}

[1] Diop, A., JACod, J. And Todorov, V. (2013). Central limit theorems for approximate quadratic variations of pure jump Itô semimartingales. Stoch. Process. Appl. 123, 839-886.

[2] JACOD, J. (2004). The Euler scheme for Lévy driven stochastic differential equations: limit theorems. Ann. Prob. 32, 1830-1872.

[3] JACOd, J. AND Protter, P. (1998). Asymptotic error distributions for the Euler methods for stochastic differential equations. Ann. Prob. 26, 267-307.

[4] JaCOD, J. AND Protter, P. (2012). Discretization of Processes. Springer, Heidelberg.

[5] Jacod, J. And Shiryaev, A. N. (2003). Limit Theorems for Stochastic Processes, 2nd edn. Springer, Berlin.

[6] Kurtz, T. G. And Protter, P. (1991). Weak limit theorems for stochastic integrals and stochastic differential equations. Ann. Prob. 19, 1035-1070. 